\title{
Inclusión de niños con TEA a través de la imple- mentación de avances tecnológicos del siglo XXI
}

\author{
Luisa Fernanda Gómez", Valentina Pérez", María Fernanda Olivera", María Alejandra Pérez"****, \\ Fransesca Santos ${ }^{* * * * * *}$, Mileidys León ${ }^{* * * * * * *}$, Ingrid Romero Lázaro*
}

\begin{abstract}
Resumen
Para este trabajo investigativo se aplicó una herramienta tecnológica orientada a dispositivos móviles (app) denominada DictaPicto, enfocada en estimular la parte visual a través de ilustraciones didácticas que sirven de apoyo para la creación de conceptos en individuos que padecen trastorno de efecto autista (TEA) -a quienes se les suele dificultar la comprensión verbal y no verbal-. Este tipo de herramientas se utilizan para facilitarles la adaptación a su entorno y, por ende, su comunicación. El objetivo general está enfocado a analizar los efectos de la aplicación en adolescentes que padecen autismo. DictaPicto ofrece beneficios para la comunicación diaria en cualquier situación para los que tienen esta condición. Se pudo concluir que haciendo uso de esta app se facilitó la lectura del contexto para favorecer la expresión y participación en el aula de clases de los estudiantes con TEA. Este tipo de estrategias facilita las relaciones interpersonales de adolescentes autistas frente a su entorno.
\end{abstract}

Palabras clave: autismo, comunicación, tecnología, trastorno

\begin{abstract}
For this research, a technological tool aimed at mobile devices (app) called DictaPicto was applied, focused on stimulating the visual part through didactic illustrations that serve as a support for the creation of concepts in individuals suffering from autistic effect disorder (ASD) -who often have difficulties to understand verbal and non-verbal messages-. These types of tools are used to facilitate their adaptation to their environment and, therefore, their communication. The overall objective is to analyze the effects of the application on adolescents suffering from autism. DictaPicto offers benefits for daily communication in any situation for those who have this condition. It was concluded that using this app facilitated the reading of the context to encourage expression and participation in the classroom of students with ASD. This type of strategy facilitates the interpersonal relationships of autistic adolescents in their social environments.
\end{abstract}

Keywords: Autism, Communication, Disorder, Technology

\footnotetext{
* Corporación Universitaria del Caribe (Cecar). Contacto: luisa.gomez@cecar.edu.co

** Corporación Universitaria del Caribe (Cecar). Contacto: valentina.perez@cecar.edu.co

*** Corporación Universitaria del Caribe (Cecar). Contacto: maría.olivera@cecar.edu.co

**** Corporación Universitaria del Caribe (Cecar). Contacto: maría.perez@cecar.edu.co

***** Corporación Universitaria del Caribe (Cecar). Contacto: fransesca.santos@cecar.edu.co

****** Corporación Universitaria del Caribe (Cecar). Contacto: mileidis.leon@cecar.edu.co

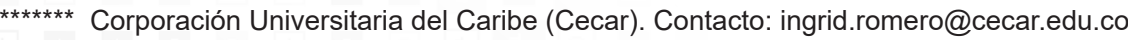




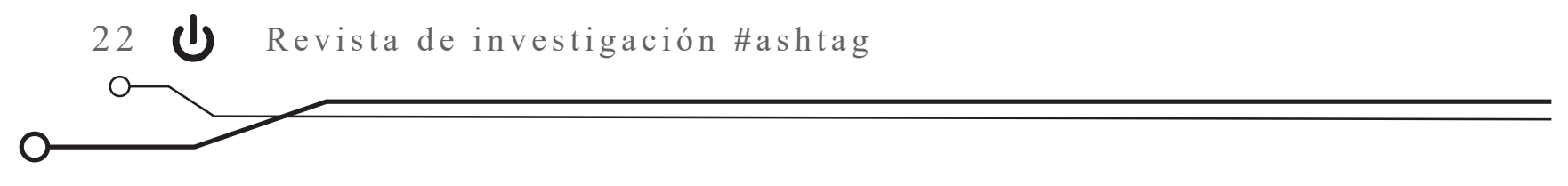

\section{Introducción}

El TEA afecta el neurodesarrollo de los niños en sus primeros años de vida, dificulta la comprensión del lenguaje y la interacción con el entorno, y presenta limitaciones en el espacio de la comunicación. En búsqueda de estrategias y con el ánimo de aprovechar los avances tecnológicos, se hará uso de los CTS (Estudios de ciencia, tecnología y sociedad) para beneficio de las personas que padecen este trastorno.

DictaPicto es una aplicación móvil que permite convertir, en tiempo real, un mensaje de voz o escrito en información visual (pictogramas o fotografías). Pensada para ayudar a los pacientes con autismo o a aquellos que usan sistemas pictográficos para comunicarse, su objetivo es mejorar el acceso a la información y facilitar la comprensión del entorno independientemente de que las personas que rodean a la persona

\section{Planteamiento del problema}

En medio de una sociedad altamente tecnificada, los adolescentes con TEA son excluidos en muchas ocasiones. Este tema se ha venido abordando desde varias perspectivas por parte de los innovadores, científicos, médicos, etc., quienes han llegado a una conclusión muy importante: las TIC son un potente recurso para las personas con TEA en varios ámbitos: educación, comunicación, ocio y tiempo libre, valoración y diagnóstico (Fernández, 2015).

Una de las tendencias desarrolladas es la implementación de apps como DictaPicto, que ayudan con TEA conozcan estos sistemas de comunicación aumentativos o alternativos. Desarrollada por BJ-Adaptaciones y Doble Equipo bajo el programa de soluciones tecnológicas de la Fundación Orange, DictaPicto permite anticipar y secuenciar actividades de la vida diaria de forma flexible y en diferentes escenarios, lo que facilita la participación y la interacción del usuario con su entorno (Belso, 2017, p. 1).

En este proyecto se implementó DictaPicto, aplicación que estimula la parte visual a través de ilustraciones didácticas que sirven de apoyo para la creación de conceptos, con el fin de facilitar la adaptación al entorno y la comunicación de los pacientes de autismo. Esta app facilita la lectura del contexto y favorece su expresión y participación en la sociedad.

a los adolescentes con TEA en la comunicación con su entorno. Desde el 2016, esta app está al alcance de todos gracias a la fundación Orange. Teniendo en cuenta lo mencionado anteriormente, se considera que el presente estudio permitirá analizar los efectos del uso de DictaPicto en adolescentes que padecen de TEA. En esta oportunidad resulta especialmente importante evaluar y conocer las diferencias que manifiesta cada paciente en sus patrones de aprendizaje con el uso de la app, que entra a desempeñar un papel fundamental a la hora de garantizar la conectividad entre los adolescentes y su entorno. 


\section{Justificación}

La razón principal por la cual estamos realizando este proyecto es ayudar a que los adolescentes con TEA alcen la voz y puedan desenvolverse más fácilmente con la sociedad, ya que uno de los grandes desafíos que afronta el adolescente con autismo es justamente la comunicación y el desarrollo de su lenguaje verbal. Se trata, pues, de encontrar y establecer vínculos entre el niño autista y las herramientas tecnológicas, y de brindarle instrumentos que le permitan desarrollar sus habilidades sociales y comunicacionales.

Se ha comprobado que la mayoría de los niños autistas tienen respuestas positivas a los estímulos visuales; eso es precisamente lo que queremos comprobar con nuestro proyecto. Para esto, recurrimos a la app DictaPicto, creada por la fundación Orange. Esta institución es especialista en los niños con TEA, hecho que nos llevó a elegir su herramienta tecnológica para el desarrollo del proyecto.

El fin de este proyecto es poder ayudar a estos adolescentes con TEA a mejorar su comunicación, su comprensión y su acceso a la información. Con ella intentamos ampliar su vocabulario al apoyarnos en los campos semánticos asociados a las acciones que ejecutan diariamente, y también mejorar la posibilidad de plantearles preguntas. Algo realmente innovador es que esta aplicación convierte en tiempo real y de forma deslocalizada el lenguaje oral, la voz, en información visual. Al respecto, Goldsmith y Le Blanc (2004) afirman que el pertinente uso de tecnologías para perfeccionar e incitar especialmente la comunicación nuestros niños con TEA se ha elevado en los últimos períodos de forma exponencial. Estas herramientas en hilos terapéuticos facilitan una generalización de la conducta hacia contextos originarios del niño.

El objetivo general está enfocado en analizar los efectos de la aplicación en adolescentes que padecen autismo. Para ello, se establecieron los siguientes objetivos específicos:

- Facilitar el acceso a la información de las personas que rodean al adolescente autista con relación a su entorno a través de una app.

- Explicar la importancia que reviste llevar a cabo una buena estimulación en un adolescente con autismo.

- Ayudar a la estimulación de un adolescente con autismo con la implementación de una app.

con base en el aislamiento social de los pacientes. Le dio el nombre de autismo infantil. Un año después, Hans Asperger, en Alemania, describió pacientes similares y nombró al trastorno como psicopatía autística (Álvarez y Camacho, 2010, p. 22). 


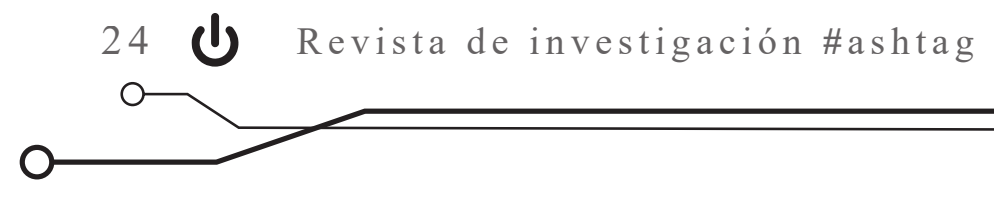

El autismo se concibe como una alteración del neurodesarrollo en la que se ve comprometido el desarrollo comunicativo, emocional y simbólico del niño, así como su capacidad de establecer relaciones con los adultos y los objetos. También afecta la intersubjetividad, es decir, el campo afectivo compartido en el que se lleva a cabo la comunicación interpersonal (Belinchón y Rivière, 1981).

El trabajo “Nuevas tecnologías: puentes de comunicación en el trastorno del espectro autista (TEA)" de Guido Guzmán, Natalia Putrino, Felipe Martínez y Nicolás Quiroz (2017), presenta una conceptualización del trastorno del espectro autista (TEA), describe las terapias validadas, continua con los estudios sobre diversas tecnologías aplicadas en el tratamiento de la enfermedad y con la importancia de su uso en un ambiente terapéutico. Finalmente, refiere la experiencia de

\section{Tipo de investigación}

Con base en el objetivo general de este proyecto, se desarrolló una investigación descriptiva que busca especificar las propiedades, características y perfiles de personas, grupos, comunidades, procesos, objetos o cualquier otro fenómeno que se someta a un análisis (Hernández-Sampieri,

\section{Técnicas de recolección de información}

La técnica seleccionada fue la entrevista, ya que está dada a la recolección de información oral. En este proyecto, que va dirigido hacia la investigación de las dificultades que presentan los adolescentes en la parte comunicativa, fue de

\section{Población}

Para este proyecto se escogió una muestra de 3 adolescentes entre los 10 y los 19 años de la Institución Educativa Francisco José de Caldas, en un desarrollo que tuvo en cuenta las consideraciones pertinentes para ser aplicadas en personas con TEA.

En 1943, el psiquiatra Leo Kanner realizó la primera clasificación del autismo. En el experimento atrás referido, describió las conductas de cada uno, especialmente aquellas que llamaron más su atención para efectos de la investigación. A pesar de las indagaciones realizadas durante más de medio siglo por Kanner y otros autores, el autismo sigue ocultando su origen y gran parte de su naturaleza, y supone retos y dificultades para su tratamiento efectivo. Después de más cincuenta años de investigación, por fin este trastorno fue reconocido oficialmente en la $3^{\text {ra }}$ edición del manual diagnóstico y estadístico de los trastornos mentales de la asociación americana de psiquiatría (Palomo, 2017).

2017). En este proyecto se hizo un diseño de campo de tipo cualitativo que implementó la entrevista como técnica de recolección de información. Por último, el propósito de esta investigación es aplicada porque se le estará aplicando a una población objeto de estudio.

vital importancia hacer uso de esta técnica también con los padres de familia porque permite acceder a la información necesaria para implementar esta app en beneficio de los adolescentes.

Corozal (Sucre). Esta muestra fue escogida a criterio personal del investigador, ya que se encontró que en esa institución hay adolescentes con TEA. 


\section{Fases metodológicas}

Tabla 1. Fases metodológicas

\begin{tabular}{|c|c|c|}
\hline Fase & Objetivo & Actividad \\
\hline I & 1 & $\begin{array}{l}\text { - Investigar todo sobre la } \operatorname{app} \text { (DictaPicto). } \\
\text { - } \quad \text { Discutir si se puede utilizar la app DictaPicto en adolescentes con TEA. } \\
\text { - Dar a conocer la app a un adolescente con TEA. }\end{array}$ \\
\hline II & 2 & $\begin{array}{l}\text { - Dar las respectivas charlas para dar a conocer la aplicación a los } \\
\text { adolescentes y sus acudientes. }\end{array}$ \\
\hline III & 3 & $\begin{array}{l}\text { - Poner en práctica la app en los adolescentes para llevar a cabo una } \\
\text { buena estimulación. }\end{array}$ \\
\hline IV & 4 & $\begin{array}{l}\text { - Reunir al acudiente o persona adulta responsable con el adolescente } \\
\text { para mostrar los resultados alcanzados hasta el momento. } \\
\text { - Implementar la app en el aula de clases para mejorar la comunicación. }\end{array}$ \\
\hline
\end{tabular}

Fuente: elaboración propia

\section{Resultados}

\section{Desarrollo del proyecto}

\section{Fase I}

Como parte de una primera actividad, se investigó en páginas de internet sobre aplicaciones innovadoras relacionadas con el TEA para tratar nuestro planteamiento del problema. En este proceso encontramos DictaPicto, a nuestro juicio, la mejor opción, ya que nos brindaba todo lo que necesitábamos y se ajustaba a nuestro diagnóstico.

Gracias a las investigaciones realizadas, se logró conceptualizar la importancia de la aplicación de esta app en los adolescentes con TEA y evidenciar de la manera más significativa cómo brinda un aporte a sus vidas, ya que, por su condición, no tienen la misma interpretación cognitiva y comportamental sobre la vida que los rodea, hecho que los aísla y les restringe muchas oportunidades, incluida la de tener una mejor comunicación con las personas más cercanas. Cabe resaltar el propósito por el cual fue creada la app: permitir de la forma más fácil que los pacientes con TEA puedan tener una mejor comunicación con el mundo que los rodea.

De acuerdo con las investigaciones realizadas, se pudo evidenciar los efectos positivos de la implementación de esta herramienta con los jóvenes, ya que esta les brinda una mejor comunicación, y les muestra de manera creativa y responsable actividades pensadas para su situación. También se les permitió a los docentes del aula conocer e interactuar con esta app con el fin 


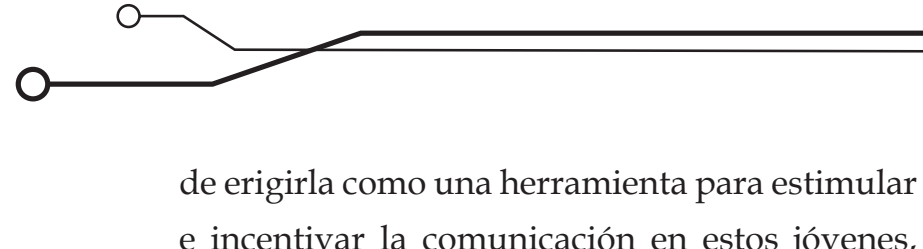
e incentivar la comunicación en estos jóvenes, cuyo reto principal es buscar una mejor interpretación de su lenguaje verbal y comportamental. Así, se puso en práctica esta app con un joven autista. De esta experiencia pudimos constatar la apreciación por parte de los docentes y la forma en que este joven dio sus primeros pasos de mejoría. En síntesis, se demostró una vez más que sí es posible que ellos puedan de una forma sencilla y entretenida tener una estimulación que los ayude a salir de la burbuja en la que se encuentran, lograr una mejor comunicación y, por supuesto, ser comprendidos de manera asertiva.

Como parte de una segunda actividad, se descargó la aplicación en nuestros celulares. Revisamos cada herramienta que brinda la app y encontramos que todo lo que esta ofrece fue llamativo para la población objeto de estudio.

Figura 1. Dictapicto: categorías

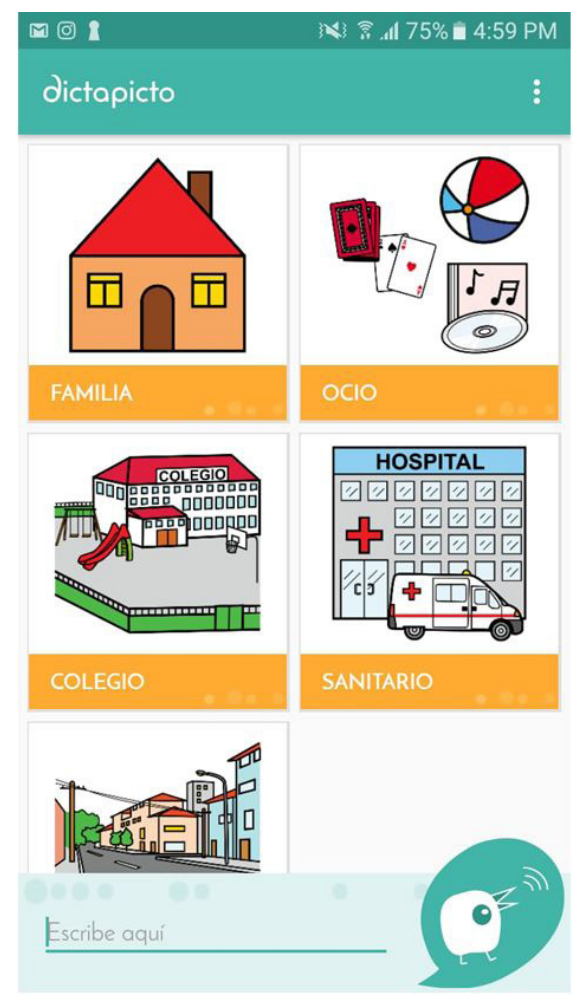

Fuente: elaboración propia
Figura 2. Dictapicto: tareas

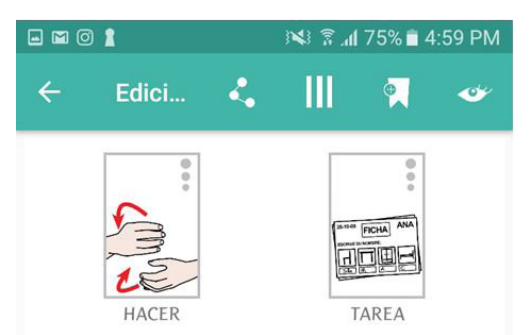

Fuente: elaboración propia

Como parte de una tercera actividad, se contó con la presencia de un adolescente con TEA. Se le dio a conocer al adolescente la app para ver qué reacción mostraba. Los resultados obtenidos fueron muy positivos, ya que, durante su interacción, este sintió conexión con la app y cada vez trataba de comunicarse más con ella.

\section{Fase II}

Como parte de una primera actividad, se organizó una charla con la intención de que los acudientes de los jóvenes con TEA conocieran la app. Esto fue benéfico para estas personas porque conocieron una nueva forma de ayudar a los jóvenes y de interactuar con ellos, y de permitirles, además, mirar la vida desde otra perspectiva, ya que ellos interactúan de una manera creativa y segura. Desde que los acudientes conocieron la $a p p$, se mostraron interesados por experimentar con los chicos. Después de unos días, los pacientes manifestaron los resultados esperados. 


\section{Fase III}

Como parte de la actividad propia de esta fase, y con ayuda de celulares, se mostró a los adolescentes la app y se les explicó su funcionamiento: primero, se habla (se dice alguna palabra o frase); luego, tienen que ver las imágenes en que

\section{Fase IV}

Como parte de una primera actividad, después de una semana de haber presentado a los adolescentes la app, se solicitó a los padres que asistieran al aula de clase para presentar los resultados obtenidos. Ellos pudieron observar cómo los adolescentes se mostraban más abiertos al momento de comunicarse, por lo que quedaron satisfechos al ver los efectos positivos del uso de esta herramienta. Posteriormente, se plantearon algunas recomendaciones a la institución con respecto a la app, ya que esta tiene la facilidad de usarla en tabletas digitales. Se les propuso que descargaran en cada tableta la app para que los adolescentes tuvieran la oportunidad de tener acceso a ella y así, de alguna forma, mejorar su se convierten las palabras o frases. El resultado fue muy alentador, ya que ellos mismos manifestaron que se sentían muy bien y que era divertido observar las imágenes.

comunicación. En la institución estuvieron de acuerdo con la propuesta.

Finalmente, tal como se puede observar en las figuras 3, 4, 5, 6 y 7, los acudientes brindaron sus opiniones con respecto a los resultados de sus hijos e indicaron que observaron avances con la implementación de la app en la vida cotidiana de los jóvenes. Con ello, otorgaron el permiso al colegio para que pueda trabajarla con ellos, ya que estos manifestaron su deseo de garantizar una mejor calidad educativa. En efecto, esta herramienta representa una oportunidad de mejora en la comunicación y actitud asertiva.

Figura 3. Encuesta de satisfaccion a padres de familia (seccion 1)

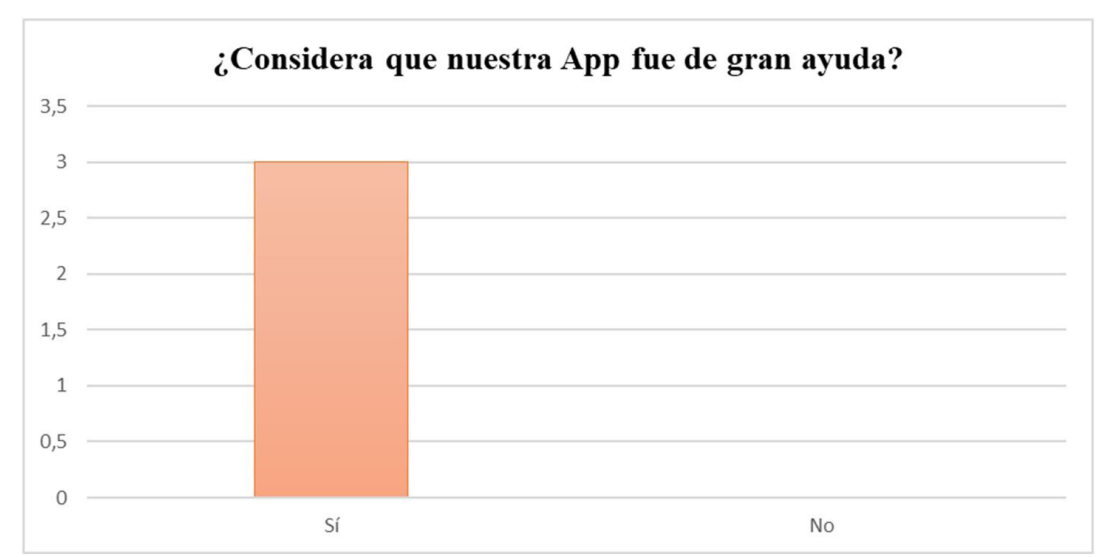

Fuente: elaboración propia 
$28 \bigcup$ Revista de investigación \#ashtag

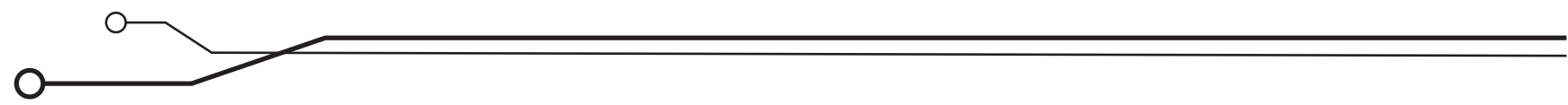

Figura 4. Encuesta de satisfaccion a padres de familia (seccion 2)

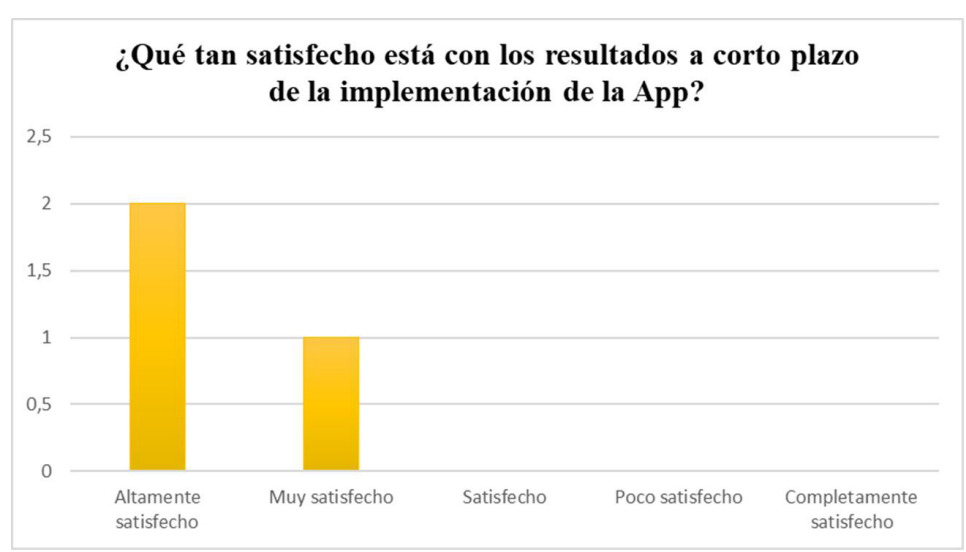

Fuente: elaboración propia

Figura 5. Encuesta de satisfaccion a padres de familia (seccion 3)

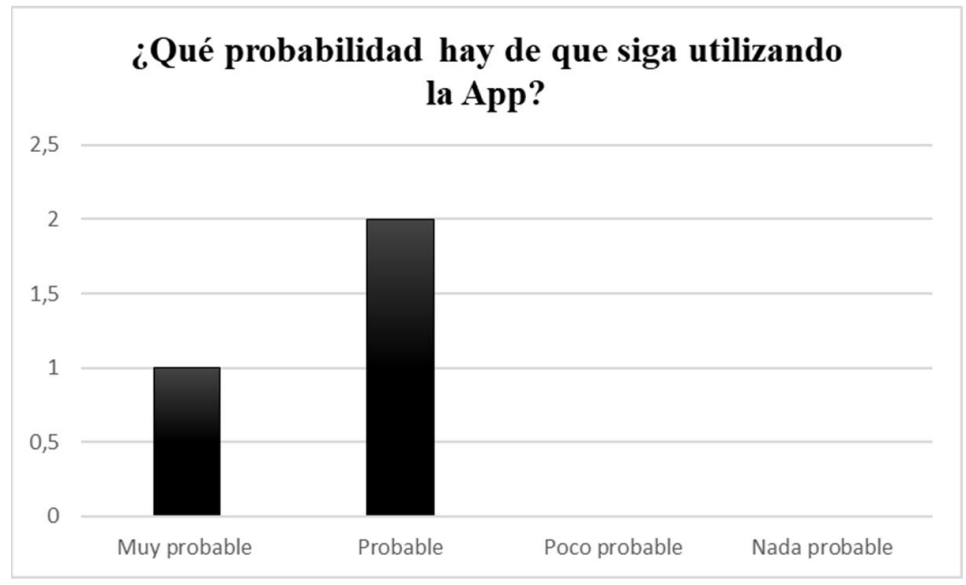

Fuente: elaboración propia

Figura 6. Encuesta de satisfaccion a padres de familia (seccion 4)

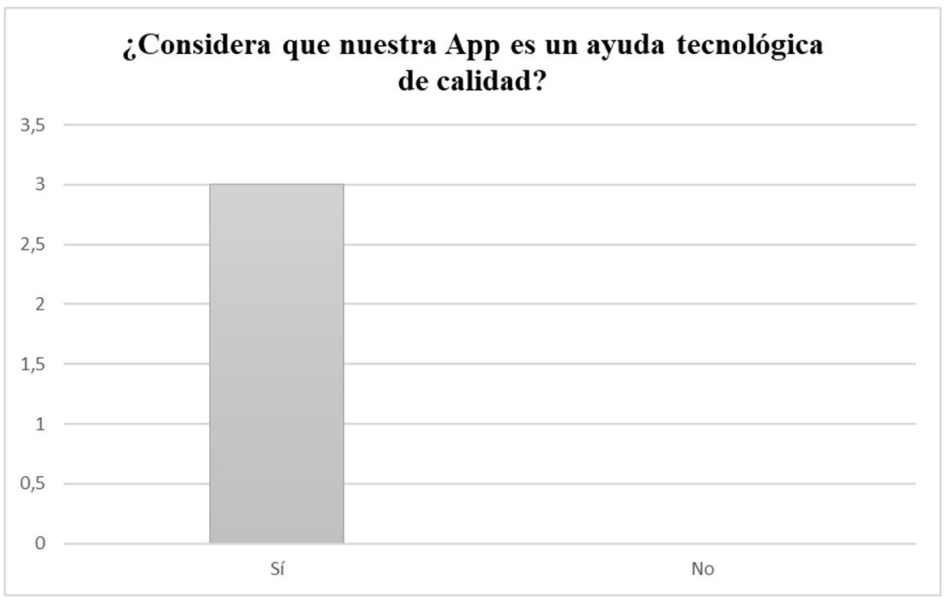

Fuente: elaboración propia 
Figura 7. Encuesta de satisfaccion a padres de familia (seccion 5)

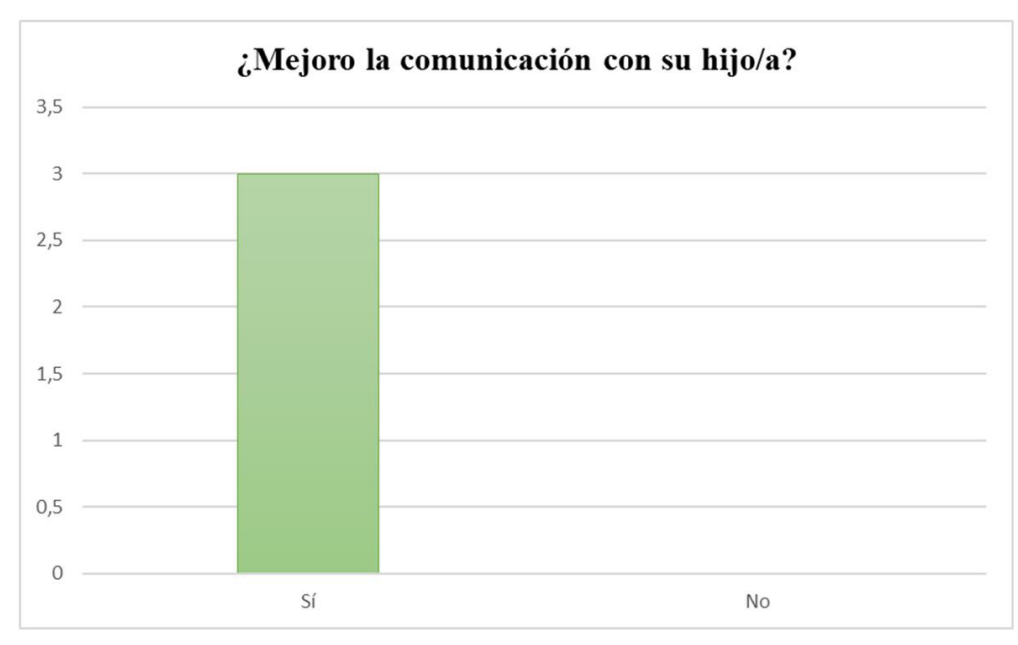

Fuente: elaboración propia

\section{Conclusiones}

Como resultado final, se obtuvo un análisis de los efectos de la implementación de una app de entrenamiento para jóvenes que padecen TEA. De allí se pudo concluir que al hacer uso de herramientas tecnológicas como DictaPicto, orientadas a mejorar la expresión y las habilidades comunicativas de los adolescentes con esta patología, se contribuye a cualificar las condiciones de los individuos en lo que refiere a las habilidades de lectura y reconocimiento de situaciones cotidianas relacionadas con el contexto real, y a que desarrollen una mejor interpretación del lenguaje verbal y comportamental. En esencia, esta aplicación los incentiva a entablar comunicación con su medio, probablemente el reto más importante que enfrentan. Sin embargo, es apropiado incluir a un profesional en el área para que realice un acompañamiento continuo.

Los adolescentes con TEA responden de manera positiva a los estímulos visuales que suministra la aplicación DictaPicto, herramienta utilizada por su comprobada efectividad en tratamientos de pacientes con TEA. Así, como resultado del proceso investigativo, se observó una mejora en los procesos comunicativos de la población objeto de estudio con sus familiares más cercanos, sus compañeros de clase y, en general, con su entorno. A partir de ello, se infiere que los adolescentes con TEA atienden de manera positiva a usar herramientas tecnológicas y a incorporarlas gradualmente en su vida cotidiana. Esta es una forma diferente de aprender que, a su vez, les brinda entretenimiento, genera efectos positivos en sus procesos comunicativos y les garantiza ser comprendidos de manera asertiva. Esto se pudo evidenciar a través de los datos obtenidos, valorados en alta satisfacción y respuesta positiva, lo que invita a seguir utilizando la tecnología como herramienta facilitadora en los procesos cognitivos y psicosociales. 


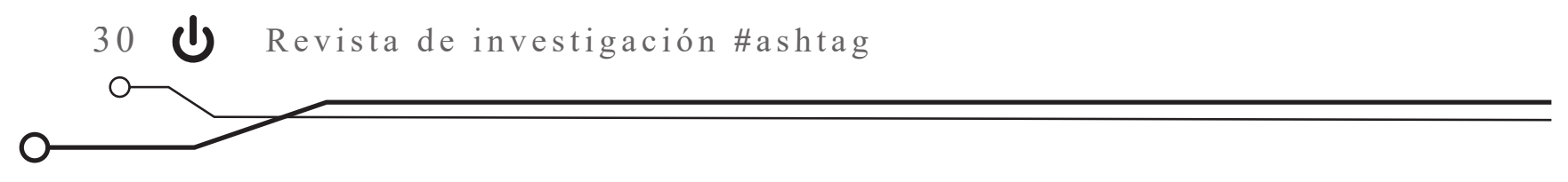

\section{Referencias}

Álvarez, I. y Camacho, I. (2010). Bases genéticas del autismo. Acta Pediátrica de México, 31(1), 22-28. Recuperado de https://www.medigraphic.com/pdfs/actpedmex/apm-2010/ apm101g.pdf

Belinchón M, Rivière A. (1981). El lenguaje autista desde una perspectiva correlacional. Estudios de Psicología, 5 y 6, 65-66. Recuperado de https://sid.usal.es/idocs/F8/ART11316/ lenguaje_autista_perspect_correlacional.pdf

Belso, J. y Bellver, A. (2017). Dictapicto. Aula abierta [recurso en línea]. Recuperado de http:// aulaabierta.arasaac.org/dictapicto-0-inicio

Fernández, L. (2015). Introducción de la tecnología como recurso para la enseñanza y el aprendizaje del alumnado con trastorno del espectro autista [trabajo de grado]. Universidad de Santiago de Compostela, España. Recuperado de https://minerva.usc.es/xmlui/bitstream/handle/10347/14553/TFG1415_Fern\%C3\%A1ndez_L\%C3\%B3pezValeiras_Luisa_P_X.pdf?sequence $=4 \&$ is Allowed $=y$

Goldsmith, T. y Le Blanc, L. (2004). Use of technology in Interventions for Children with Autism. Journal of Early and Intensive Behavior Intervention, 1(2), 166-178. Recuperado de http:/ / dx. doi.org/10.1037/h0100287

Guzmán, G., Putrino, N., Martínez, F. y Quiroz, N. (2017). Nuevas tecnologías: puentes de comunicación en el trastorno del espectro autista (TEA). Terapia Psicológica, 35(3), 247-258. Recuperado de https://scielo.conicyt.cl/scielo. php?script=sci_abstract\&pid=S0718-48082017000300247\&lng=es\&nrm=iso

Hernández-Sampieri, R. (2017). Fundamentos de investigación. Ciudad de México: McGraw Hill.

Palomo, R. (2017). Autismo. Madrid: Alianza Editorial. 